\title{
Le droit suisse des sociétés en 2020 : Modifications législatives
} et jurisprudence

Cette contribution propose un aperçu des principales modifications législatives intervenues durant l'année 2020 en droit suisse des sociétés, ainsi que de la jurisprudence du Tribunal fédéral rendue pendant la même période. La section consacrée aux modifications législatives (chiffre I.) présente en particulier, de manière succincte, la modification du droit de la société anonyme [SA] adoptée le 19juin 2020. Les résumés de jurisprudence font l'objet de la section suivante (chiffre II.); les arrêts sont classés par forme de société. Après la présentation des arrêts publiés au recueil des ATF, les arrêts sont classés par ordre chronologique.

I. Modifications législatives

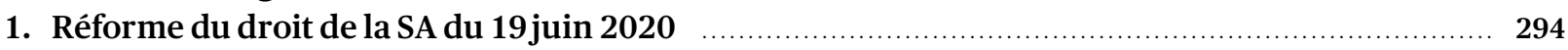

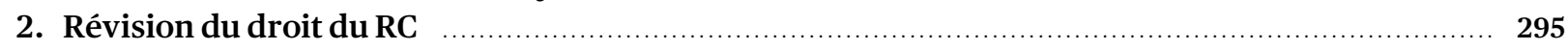

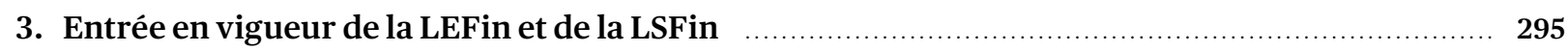

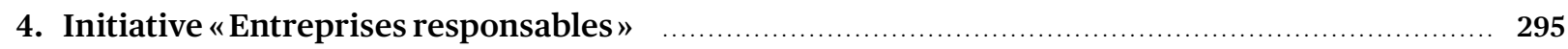

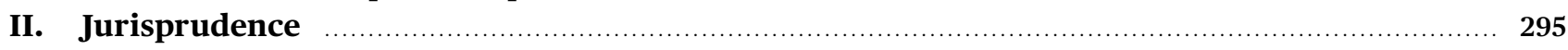

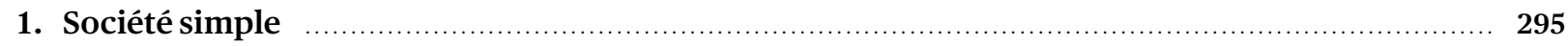

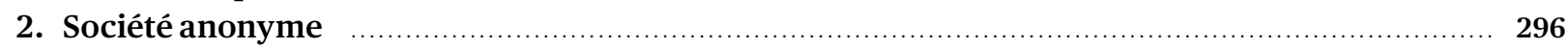

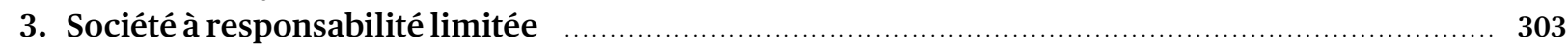

Citation:

DAMIANO CANAPA / ARTHUR GRISONI /

MARINE ANTONOFF, Le droit suisse des sociétés en 2020: Modifications législatives et jurisprudence,

sui generis 2021, S. 293

Damiano Canapa, Professeur à l'Université de Lausanne, LL.M. (Bruges), LL.M. (Yale). Arthur Grisoni, MLaw, Assistant doctorant à l'Université de Lausanne. Marine Antonoff, MLaw, Assistante doctorante à l'Université de Lausanne. Les auteurs remercient Me Cédric Ballenegger pour sa relecture attentive de leur contribution.

URL: sui-generis.ch/193

DOI: https://doi.org/10.21257/sg.193

Ce(tte) œuvre est mise à disposition selon les termes de la Licence Creative Commons Attribution - Partage dans les Mêmes Conditions 4.0 International. 


\section{Modifications législatives}

\section{Réforme du droit de la SA du 19juin $2020^{1}$}

1 Après deux messages ${ }^{2}$, la scission de la révision en plusieurs projets ${ }^{3}$, l'ajout de nouvelles thématiques en cours de travaux ${ }^{4}$ et de longues années de débats parlementaires ${ }^{5}$, l'Assemblée fédérale a finalement adopté, le 19juin 2020, la réforme du droit de la $\mathrm{SA}^{6}$. En raison de son format, cette contribution expose uniquement les évolutions les plus marquantes de la modification, la plus importante du droit de la SA depuis celle du 4 octobre $1991^{7}$. Si la date de l'entrée en vigueur de l'essentiel de la réforme est inconnue à ce jour (1 ${ }^{\text {er }}$ septembre 2021), elle devrait vraisemblablement intervenir au début ou dans le courant de l'année 2023.

2 Sil'unité du droit de la SA demeure dans l'ensemble préservée, il n'en demeure pas moins que les différences entre SA privées et SA publiques s'accentuent; l'exemple le plus marquant est représenté par les art. $732 \mathrm{ss} \mathrm{nCO}$ («Rémunérations dans les sociétés dont les actions sont cotées en bourse $»)^{8}$. Les conditions d'exercice de nombreux droits d'actionnaires sont à présent différenciées : des seuils différents s'appliquent s'agissant de la convocation d'assemblées générales [AG] extraordinaires (art.699 al. 3 nCO), de l'inscription d'un objetà l'ordre du

1 Code des obligations (Société anonyme), Modification du 19juin 2020 (RO 2020 4005).

2 Message du 21 décembre 2007 concernant la révision du code des obligations (Droit de la société anonyme et droit comptable; adaptation des droits de la société en nom collectif, de la société en commandite, de la société à responsabilité limitée, de la société coopérative, du registre du commerce et des raisons de commerce) (FF 2008 1407); Message du Conseil fédéral du 23 novembre 2016 concernant la modification du code des obligations (Droit de la société anonyme) (FF 2017 353).

3 La réforme du droit comptable(RO 2012 6679), initialement intégrée à la réforme du droit de la SA, Message du concernant la révision du code des obligations (2007) (n. 2), p.1421), a été adoptée le 23 décembre 2011 (RO 20126679 ).

4 Suiteà l'adoption, le 3 mars 2013, de l'initiative populaire « contre les rémunérations abusives ", dite initiative Minder (Arrêté du 30 avril 2013 du Conseil fédéral constatant le résultat de la votation populaire du 3 mars 2013; FF 2013 2759), le Parlement a été chargé de réglementer les rémunérations dans les SA cotées en bourse.

5 Objet du Conseil fédéral 16.077 (CO. Droit de la société anonyme).

6 Au sujet de cette réforme, voir PETER FORSTMOSER/MARCEL KÜCH LER, Die Reform 2020 des schweizerischen Aktienrechts, RDS 2020 I, p. 393 ff.; HANS CASPAR VON DER CRONE, Aktienrecht, $2^{\mathrm{e}}$ éd., Berne 2020; DAMIANO CANAPA/MARLEEN BOTTERBRODT / ANTOINE SCHNEEBELI, Le droit suisse des sociétés en 2017: Modifications législatives, jurisprudence et doctrine, sui generis 2018, p. 299 ss ; DAMIANO CANAPA/ARTHUR GRISONI/LINE ELODIE DERUNGS, Le droit suisse des sociétés en 2018: Modifications législatives, jurisprudence et doctrine, sui generis 2019, p. $301 \mathrm{~s}$.; DAMIANO CANAPA / ARTHUR GRISONI / LINE ELODIE DERUNGS, Le droit suisse des sociétés en 2019: Modifications législatives, jurisprudence et doctrine, sui generis 2020 , p. 430 .

7 Code des obligations (Société anonyme), Modification du 4 octobre 1991 (RO 1992 733).

8 Ordonnance du 20 novembre 2013 contre les rémunérations abusives dans les sociétés anonymes cotées en bourse (ORab; RO 2013 4403). jour (art.699b nCO) et de l'institution d'un examen spécial (nouvelle dénomination du «contrôle spécial», $\mathrm{cf}$. art. 697a ss CO, 697d nCO).

La réforme apporte davantage de flexibilité au niveau de 3 la réglementation du capital. L'introduction de la marge de fluctuation du capital (art. 653s ss nCO), qui remplace l'augmentation autorisée, constitue l'innovation la plus importante. Elle permet, à travers une clause statutaire adoptée à la majorité qualifiée (art. 704 al.1 ch. 5 nCO), d'autoriser le conseil d'administration [CA] à modifier le capital-actions dans une certaine limite supérieure et inférieure, qualifiée de «marge de fluctuation », durant une période de cinq ans au maximum. La reprise de biens (art. $628 \mathrm{al} .2 \mathrm{CO}$ ) n'est plus réglementée, tandis que les caractéristiques essentielles de l'apport en nature, jusqu'alors réglées dans la Communication de l'OFRC du 15 août 2001 relative aux apports en nature et aux reprises de biens, sont codifiées dans la loi (art. 634 al. 1 ch. 4 nCO). Enfin, le capital-actions peut être émis dans une devise étrangère (art. $621 \mathrm{al} .2 \mathrm{nCO}$ ) et la valeur nominale de chaque action doit uniquement être supérieure à zéro (art. $622 \mathrm{al} .4 \mathrm{nCO}$ ) ; la référence à la valeur nominale minimale de 1 centime est abandonnée.

Les droits des actionnaires sont élargis. De nombreux 4 seuils permettant à une minorité d'actionnaires d'exercer leurs droits sont revus à la baisse. Tel est le cas du droit de convocation d'une AG extraordinaire, du droit d'obtenir l'inscription d'un objet à l'ordre du jour et du droit de requérir l'institution d'un examen spécial en cas de refus de l'AG, déjà évoqués supra. Dans ces trois cas, il est davantage tenu compte de l'existence d'actions à droit de vote privilégié : une minorité du capital-actions ou des voix est nécessaire pour exercer ces droits. L’on notera également l'introduction de la possibilité de verser, en cours d'exercice, des dividendes intermédiaires (art.675a nCO).

Sur le plan de la perte de capital et du surendettement, 5 un nouveau mécanisme d'annonce précoce est introduit (art. $725 \mathrm{al} .2 \mathrm{nCO}$ ) : si la SA menace de devenir insolvable, le CA est tenu de prendre des mesures ou de proposer des mesures à l'AG, si elles relèvent de sa compétence. Pour l'avis au juge, le CA, en cas de surendettement, peut renoncer à aviser le tribunal tant qu'il existe des raisons sérieuses d'admettre qu'il est possible de supprimer le surendettement, mais au plus pendant 90 jours après l'établissement des comptes, et si l'exécution des créances n'est pas davantage compromise (art. $725 b$ al. 4 ch. 2 nCO).

S'agissant des organes, l'AG peut se tenir de manière vir- 6 tuelle (art. 701d nCO), à plusieurs endroits simultanément (art. $701 a$ al. 3 nCO), voire à l'étranger (art. $701 b$ nCO). L'usage de médias électroniques permettant aux 
actionnaires n'étant pas présents sur le lieu de l'AG peut aussi être autorisé par le CA (art. 701c nCO). Les possibilités de représentation institutionnelle sont plus limitées dans les SA cotées en bourse, où seul le représentant indépendant demeure autorisé (art. 689 c nCO). Quant au CA, la présomption concernant la délégation de la gestion à une direction externe est renversée : dorénavant, à défaut de clause statutaire contraire, une telle délégation est autorisée (art. $716 b$ al. 1 nCO). Enfin, quelques nouvelles tâches incombent de manière ponctuelle à l'organe de révision [OR], tel que le contrôle des comptes intermédiaires en cas de réduction du capital-actions intervenant dans le cadre d'une marge de fluctuation du capital (art. $653 \mathrm{u}$ al. $3 \mathrm{nCO}$ ) ou de versement de dividendes intermédiaires (art. 675a al. 2 nCO).

7 Sur le plan de la politique d'entreprise, il existe des changements importants. D'une part, des quotas de représentation des sexes sont introduits dans le CA et la direction des SA cotées en bourse (art. $734 f \mathrm{nCO} ; 30 \%$ dans les CA et $20 \%$ dans les directions). En ce qui concerne le CA, des informations en lien avec l'obligation devront être fournies dans le rapport de rémunération au plus tard à compter de l'exercice qui débutera cinq ans après l'entrée en vigueur de la disposition; ce délai se monte à 10 ans pour les informations relatives à la direction ${ }^{9}$. Si les quotas ne sont pas atteints, la SA devra en expliquer les raison ("comply or explain»). Ensuite, des règles de transparence financière dans le domaine des matières premières prévoient des obligations de faire rapport sur les paiements effectués au profit de gouvernements à charge des SA actives dans certains secteurs sensibles (art. 964a ss nCO). Enfin, les possibilités de refuser un actionnaire dans les SA cotées en bourse sont élargies afin d'éviter les rachats de SA suisses par certains investisseurs étrangers (art. 685 d al. 2 nCO).

8 La réforme du droit de la SA modifie également d'autres parties du CO - notamment en droit de la Sàrl, en droit de la SCoop ou encore en droit comptable -, mais aussi d'autres actes législatifs de droit privé (CC, LFus, CPC, LP), de droit pénal (CP), de droit fiscal (LT, LIFD, LHID, LIA), de droit des assurances (LPP, LSA) et de droit économique (LPCC, LB).

\section{Révision du droit du $\mathrm{RC}^{10}$}

9 Les nouvelles dispositions du CO (art.928b et 928c) et de l'ORC instaurant une base de données centrale des

9 Cf. art. 4 des dispositions transitoires de la modification du 19juin 2020 (RO 20204005 [n.1], ch. III).

10 Code des obligations (Droit du registre du commerce), Modification du 17 mars 2017 (RO 2020 957); Message du 15avril 2015 concernant la modification du code des obligations (Droit du registre du commerce) (FF 2015 3255). personnes sont entrées en vigueur le $1^{\mathrm{er}}$ avril 2020. Les autres dispositions issues de cette révision sont entrées en vigueur le $1^{\mathrm{er}}$ janvier 2021.

\section{Entrée en vigueur de la LEFin et de la LSFin ${ }^{11}$}

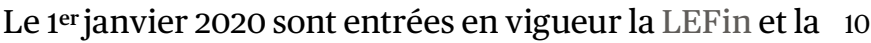
LSFin. Sur le plan du droit des sociétés, on relève que les dispositions du CO relatives au prospectus d'émission (art. 652a, 752 et 1156 CO) sont abrogées et remplacées par les art. 35 ss et 69 LSFin. La notion de représentant indépendant se réfère à celle d'établissement financier au sens de la LEFin, en plus de celle d'établissements soumis à la LB (art. $689 d$ al. 3 nCO).

\section{Initiative « Entreprises responsables"}

Le 29 novembre 2020, l'initiative populaire «Entreprises 11 responsables - pour protéger l'être humain et l'environnement " a été rejetée par une majorité des cantons ${ }^{12}$. La date de l'entrée en vigueur du contre-projet indirect adopté en parallèle à la révision du droit de la SA ${ }^{13}$ est inconnue à ce jour.

\section{Jurisprudence}

\section{Société simple}

a) Légitimation active de l'associé simple ${ }^{14}$

aa) Faits

Le 23 décembre 1993, C.A., D.A. et E.A. concluent avec B. 12 SA un bail à ferme par lequel ils cèdent l'usage des droits d'édition du journal xxx. En 2001, B. SA acquiert les droits d'édition et s'acquitte du prix de vente avec trois factures datées du 17 décembre 2001, une par associé. E.A. décède le 29 mai 2005. Le 20 août 2010, A.A., veuve et héritière de E.A., actionne B. SA en paiement devant le Pretura de Bellinzone au motif qu'une surtaxe sur le prix de vente des droits d'édition de 35\% aurait été versée à C.A. et D.A, mais non à E.A. L'action est rejetée en raison du défaut de qualité pour agir de A.A., décision qui est confirmée par le Tribunale di appello du canton du Tessin. A.A. recourt en matière civile.

11 Loi fédérale du 15juin 2018 sur les établissements financiers (LEFin RS 954.1); Loi fédérale sur les services financiers du 15juin 2018 (LSFin; RS 950.1); Message du 4 novembre 2015 concernant la loi sur les services financiers (LSFin) et la loi sur les établissements financiers (LEFin) (FF 2015 8101).

12 Rejet par 141/2 cantons alors que la majorité du peuple (50.73\%) s'était prononcée en faveur de l'initiative. Sur cette initiative, cf. Message du15septembre 2017 relatif à l'initiative populaire « Entreprises responsables - pour protéger l'être humain et l'environnement » (FF 2017 5999).

13 Objet du Conseil fédéral 16.077 (n. 5).

14 Arrêt du Tribunal fédéral 4A_25/2020 du 29juin 2020. 


\section{bb) Droit}

13 Les associés d'une société simple doivent agir conjointement en vertu de l'art. 544 al. 1 CO (consorité active nécessaire, cf. art. 70 CPC15). Si le contrat de société simple confère le droit d'agir à titre individuel, une action conjointe des associés n'est plus nécessaire; dans un tel cas, le jugement n'a pas d'effet constitutif à l'égard des autres associés. Partant, A.A. (veuve de E.A.) a la qualité pour agir si, comme elle le prétend, le contrat de société simple procure le droit d'agir à titre individuel à E.A., ce qui requiert une analyse sur le fond ${ }^{16}$.

Après avoir confirmé que C.A., D.A. et E.A. formaient une société simple et nié le droit de E.A d'agir à titre individuel, ${ }^{17}$ le Tribunal fédéral indique que la prétention de A.A. ne saurait découler d'une cession de la créance litigieuse à E.A. intervenue dans le cadre de la liquidation de la société. En effet, la société simple ne possédait pas une telle créance au moment de la dissolution. Or, selon la recourante, c'est cette cession qui aurait fondé le droit de E.A d'agir à titre individuel18 $\mathbf{~ S i ~ - ~ h y p o t h e ̀ s e ~ c o n t r o - ~}$ versée - la liquidation de la société s'était achevée par la vente et le paiement des parts sociales, la créance litigieuse aurait tout au plus été qualifiée d'actif social découvert après la liquidation. La procédure de liquidation aurait alors dû être reprise selon les règles générales découlant de l'art. 544 al. $1 \mathrm{CO}$, qui prévoient l'action en commun des associés simples ${ }^{19}$. Le recours est rejeté20.

\section{Société anonyme}

a) Action en responsabilité : légitimation active des créanciers cessionnaires des droits de la masse après radiation ${ }^{21}$

aa) Faits

15 La faillite de D. SA est prononcée le 23 avril 2013. Au cours de la procédure, la masse cède les créances en responsabilité contre les organes aux créanciers B. SA et C. SA (art. 260 LP22). Le 17 novembre 2014, la procédure som-

15 Code de procédure civile du 19 décembre 2008 (CPC; RS 272).

16 Arrêt du Tribunal fédéral 4A_25/2020 du 29juin 2020 c. 3.2 et 3.3.

17 Arrêt du Tribunal fédéral 4A_25/2020 du 29juin 2020 c. 4. à 4.5.

18 Arrêt du Tribunal fédéral 4A_25/2020 du 29juin 2020 c. 5 .

19 Arrêt du Tribunal fédéral 4A_25/2020 du 29juin 2020 c. 6.

20 Arrêt du Tribunal fédéral 4A_25/2020 du 29juin 2020 c. 7 .

21 ATF 146 III 441. Sur cet arrêt voir: FABIO ANCESCHI/MARKUS VISCHER, Bundesgericht, I. zivilrechtliche Abteilung, Urteil 4A_19/2020 vom 19. August 2020, A. gegen B. AG und C. AG, Aktienrechtliche Verantwortlichkeit; Löschung der Gesellschaft im Handelsregister; Auswirkungen auf die Abtretungsgläubiger nach Art. 260 SchKG, PJA 2020, p. 1619 ss; MELANIE HUBER-LEHMANN, Bundesgericht, I. zivilrechtliche Abteilung, Urteil vom 19. August 2020, BGer 4A_19/2020 (zur amtlichen Publikation vorgesehen), PCEF 52/2020, p. 325 ss; THIEMO STURNY / DZEVRIJE ZENDELI, Gesetzgebung und Rechtsprechung, RR-VR 6/2020, p. $11 \mathrm{~s}$.

22 Loi fédérale du 11 avril 1889 sur la poursuite pour dettes et la faillite (LP; RS 281.1) maire de faillite est clôturée et D. SA est radiée du RC. Le 17 novembre 2015, les créanciers cessionnaires ouvrent action en responsabilité contre A., administrateur de la société. Par décision du 20 mars 2017, Le Bezirksgericht de Münchwilen rejette l'action pour défaut de légitimation active, D. SA ayant été radiée du RC. Le 18 avril 2017, la société est réinscrite au RC à des fins de liquidation. Le 13juillet 2017, considérant la réinscription de la société comme un vrai novum rétablissant la légitimité active des cessionnaires, l'Obergericht du canton de Thurgovie admet l'appel de ces derniers et renvoie la cause au Bezirksgericht pour nouvelle décision au fond. Le Bezirksgericht puis, sur appel, l'Obergericht, font droit à l'action des créanciers et condamnent A. au paiement de dommages-intérêts. A. recourt en matière civile auprès du Tribunal fédéral.

\section{bb) Droit}

Le Tribunal fédéral examine si les créanciers admis à 16 l'état de collocation ${ }^{23}$ et ayant obtenu la cession des droits de la masse (art. 260 LP) antérieurement à la radiation d'une SA ont la qualité pour agir après la radiation de la société24. Le Tribunal fédéral confirme qu'une SA cesse d'exister à compter du jour de sa radiation du RC (effet constitutif de la radiation) ${ }^{25}$, mais la radiation n'éteint pas les créances non liquidées ${ }^{26}$. Les droits patrimoniaux découverts après la radiation sont des res nullius ; cela ne signifie toutefois pas que les créances restent sans maître et que l'art. $718 \mathrm{CC}^{27} \mathrm{~s}$ 'applique aux choses. La réalisation et la distribution du produit de réalisation de telles créances s'opèrent en vertu de l'art 269 LP, à l'exclusion des art. 57 et $466 \mathrm{CC}^{28}$. Cette procédure de réouverture de la faillite ne nécessite pas la réinscription de la SA aussi longtemps que l'administration de la masse ne fait pas valoir activement des créances ou d'autres actifs ${ }^{29}$.

23 Sur la nécessité, pour le créancier intentant une action en responsabilité, d'être préalablement admis à l'état de collocation, voir également ATF 136 III 322 c. 4.7.

24 On ne saurait, in casu, appliquer par analogie l'arrêt du Tribunal fédéral 4A 384/2016 du 1 er février 2017. Le Tribunal fédéral était parvenu à la conclusion que l'action ouverte par les créanciers cessionnaires devait être rejetée au fond en raison de la radiation de la société du RC suite à la suspension de la faillite pour faute d'actifs : la prétention n'ayant alors plus de titulaire, la légitimation active des créanciers demandeurs faisait défaut. Toutefois, le facteur décisif pour refuser la légitimation active des créanciers cessionnaires, en plus de la radiation de la société, consistait en l'absence de leur admission à l'état de collocation au moment de la radiation de la société, empêchant alors la cession des droits de la masse selon l'art. $757 \mathrm{al} .2$ $\mathrm{CO}$. Tel n'est pas le cas dans la présente affaire, puisqu'une cession des droits selon l'art. 260 LP (laquelle ne peut être obtenue que par un créancier colloqué) a eu lieu avant la radiation de la société, cf. ATF 146 III 142 c. 2.1 Pour un résumé de cet arrêt, voir DAMIANO CANAPA / MARLEEN BOTTERBRODT / ANTOINE SCHNEEBELI, Le droit suisse des sociétés en 2017: modifications législatives, jurisprudence et doctrine, sui generis 2018, p. 306 s.).

25 ATF 146 III 441 c. 2.2 et 2.4 .1

26 ATF 146 III 441 c. 2.4 , 2.5.4 et 2.5.5.

27 Code civil suisse du 10 décembre 1907 (CC; RS 210).

28 ATF 146 III 441 c. $2.4 \cdot 4.1$

29 ATF 146 III 441 c. 2.4 .4 .1 et 2.5.4. 

est une institution suigeneris du droit des poursuites qui confère aux créanciers cessionnaires le droit de faire valoir, en leur nom et pour leur compte, des prétentions de la masse en faillite (mandat procédural). Seul le droit d'action est transféré : la communauté des créanciers reste matériellement titulaire des créances cédées, sans être partie aux procédures menées par les cessionnaires. La réinscription de la société n'apparaît donc pas nécessaire à la conduite du procès, la radiation n'ayant aucun impact sur le droit d'action des cessionnaires ${ }^{30}$. Exiger la réinscription de la société serait contradictoire avec la possibilité de prononcer la clôture de la faillite - qui entraîne la radiation de la SA (art.159a al. 1 let.b ORC 31) sans attendre la fin des litiges relatifs aux créances cédées (art. 95 $\left.\mathrm{OAOF}^{32}\right)^{33}$. De plus, la loi accorde la possibilité, en cas de réouverture de la faillite, de procéder à la cession de certains droits «douteux» en vertu de l'art. $260 \mathrm{LP}$ (art. 269 al. 3 LP). Comme la réouverture de la faillite ne nécessite pas la réinscription de la société au $\mathrm{RC}$, la radiation de cette dernière ne saurait donc empêcher les créanciers de faire valoir en justice les créances qui leur ont été cédées en vertu de l'art. 260 LP à une époque antérieure à ladite radiation ${ }^{34}$. La radiation d'une société avant l'issue des procédures afférentes aux créances cédées est conforme à la LP et constitue une pratique répandue ${ }^{35}$.

18 In casu, la radiation de la D. SA - intervenue postérieurement à la cession des créances selon l'art. 260 LP - ne saurait affecter la légitimation active des cessionnaires ni l'existence des créances, dont le recouvrement ne nécessite pas la réinscription de la SA au $\mathrm{RC}^{36}$. Le Tribunal fédéral rejette le recours ${ }^{37}$.

\section{b) Responsabilité de la société pour acte illicite commis pas ses organes ${ }^{38}$ \\ aa) Faits}

19 Le 14 juin 2007, A. ouvre un compte bancaire auprès de la banque C. La gestion de ce compte est confiée par A. à la société B. SA par contrat de gestion de fortune. D., administrateur avec signature individuelle de B. SA, est

\footnotetext{
30 ATF 146 III 441 c. 2.5.1 et 2.6.

31 Ordonnance sur le registre du commerce du 17 octobre 2007 (ORC, RS 221.411).

32 Ordonnance sur l'administration des offices de faillite du 13juillet 1911 (OAOF ; RS 281.32).

33 ATF 146 III 441 c. 2.5.3. Le Tribunal fédéral mentionne la possibilité de distribuer aux créanciers, après radiation de la SA, le produit excédentaire des actions ouvertes selon l'art. 260 LP.

34 ATF 146 III 441 c. 2.5.5.

35 ATF 146 III 441 c. 2.7.

36 ATF 146 III 441 c. 2.7 et 2.8. Le Tribunal fédéral réserve le cas où la réinscription est nécessaire pour que la liquidation de la faillite de la société radiée puisse être terminée (art. $935 \mathrm{al} .2 \mathrm{ch} .4 \mathrm{CO}$ ).

37 Arrêt du Tribunal fédéral 4A_19/2020 du19août 2020 c. 4., non publiê à l'ATF 146 III 441.

38 Arrêt du Tribunal fédéral 4A_613/2018 du 17janvier 2020.
}

désigné comme gestionnaire du compte. En 2010, D. falsifie trois ordres de paiement concernant le compte de A. en imitant la signature de cette dernière. Ces ordres sont exécutés par la banque C., faisant subir un dommage à A. Le 5janvier 2017, A. - considérant que les actes litigieux de D. sont imputables à B. SA - actionne B. SA en paiement. Le Tribunal de première instance du canton de Genève déboute $\mathrm{A}$. et la Chambre civile de la Cour de justice rejette son appel. A. interjette un recours en matière civile au Tribunal fédéral.

\section{bb) Droit}

L'art. 722 CO institue une responsabilité de la SA pour les 20 actes illicites de ses organes commis dans la gestion des affaires de la SA ${ }^{39}$, à savoir lorsqu'un acte «entre, par un rapport fonctionnel, dans le cadre général des attributions de l'organe». En cas d'acte illicite, un tel rapport existe lorsque l'acte entre dans le cadre général de l'activité de l'organe; il importe peu que l'organe agisse dans son intérêt personnel (et non dans celui de la société). La SA doit ainsi supporter le risque qu'un de ses organes falsifie une signature. En revanche, si un acte est commis par l'organe «à l'occasion de l'exercice de ses attributions" ou à titre privé, la responsabilité de la SA ne saurait être engagée ${ }^{40}$.

B. SA était chargée par contrat de gestion de fortune des 21 relations avec la banque C. ; elle adressait directement des ordres à la banque. Les ordres falsifiés transmis par D. entraient donc dans le cadre de ses attributions d'organe de B. SA «par un rapport fonctionnel». Il importe peu que des ordres aient été falsifiés ou qu'ils n'aient pas été rédigés sur un papier comportant l'en-tête de B. SA. La responsabilité pour actes illicites de l'art. $722 \mathrm{CO}$ est une responsabilité envers le tiers (in casu A.), non envers la banque. À la différence de la représentation de la SA au sens de l'art. $718 \mathrm{CO}$ - laquelle exige que l'organe agisse au nom de la SA pour que cette dernière soit liée -, la responsabilité de l'art. $722 \mathrm{CO}$ nécessite simplement que l'organe agisse dans le cadre de ses attributions. D. aurait dû se présenter comme organe de B. SA pour engager contractuellement cette dernière vis-à-vis de la banque C., non pour engager la responsabilité délictuelle de la sociétét1.

A. dispose d'un concours d'action: contre D., responsable 22 direct (art. 41 ss CO, art. 55 al. 3 CC), contre B. SA, dont D. était l'organe (art. $722 \mathrm{CO}$ ), et contre la banque C. (en lien avec la vérification de l'ordre et de sa signature). La cour cantonale n'avait donc pas à examiner le rapport existant

39 Sur la notion d'organe au sens de l'art. 722 CO, cf. Arrêt du Tribunal fédéral 4A 613/2018 du 17janvier 2020 c. 3.2.1. Dans le cas présent, la qualité d'organe de B. SA de D. n'est pas contestée, cf., Arrêt du Tribunal fédéral 4A 613/2018 du 17janvier 2020 c. 3.3.1.

40 Arrêt du Tribunal fédéral 4A_613/2018 du 17janvier 2020 c. 3.2.2. 41 Arrêt du Tribunal fédéral 4A_613/2018 du 17 janvier 2020 c. 3.3.2. 
entre les fautes de B. SA et de la banque $\mathrm{C}^{42}$. Le recours est admis et la cause est renvoyée à la Cour cantonale ${ }^{43}$.

\section{c) Carence dans l'organisation de la société ${ }^{44}$ aa) Faits} SA, qui détient la totalité des actions de C. SA et de D. SA. En raison d'un conflit majeur et persistant entre E. et A., les trois sociétés ne parviennent plus à prendre la moindre décision. Elles n'ont plus d'administrateur depuis les AG ordinaires du 8 décembre 2017 portant sur l'exercice de 2015, et les sociétés ont cessé leurs activités fin 2015. Le 9 mars 2018, le RC du canton de Genève requiert du Tribunal de première instance qu'il prenne les mesures nécessaires en raison de carences dans l'organisation des trois sociétés ( $\operatorname{art} .731 b \mathrm{CO}$ ) et désigne un commissaire en vue de les représenter. A. conclut à ce que soit ordonnée la vente aux enchères des actions de $\mathrm{B}$. SA, tandis que $\mathrm{E}$. et les trois SA concluent à la dissolution des sociétés. Le 7 janvier 2019, le Tribunal de première instance du canton de Genève ordonne la vente aux enchères des actions de B. SA. Le 30 août 2019, la Cour de justice, sur appel de E., réforme le jugement et ordonne la dissolution et la liquidation des trois sociétés. A. recourt en matière civile.

\section{bb) Droit}

Le juge dispose d'un pouvoir d'appréciation lorsqu'il examine les mesures pouvant être envisagées en cas de carence; l'art. 731b CO n'énumérant pas exhaustivement ces mesures ${ }^{45}$. Le juge doit néanmoins respecter le principe de la proportionnalité, la dissolution constituant l'ultima ratio ${ }^{46}$.

La procédure de l'art. $731 b$ CO vise à régler aussi rapidement que possible la situation de blocage, non à résoudre le conflit des actionnaires ${ }^{47}$. Il n'est pas question de déter-

42 Arrêt du Tribunal fédéral 4A_613/2018 du 17janvier 2020 c. 3.3.2.

43 Arrêt du Tribunal fédéral 4A_613/2018 du 17janvier 2020 c. 4.

44 Arrêt du Tribunal fédéral 4A_499/2019 du 25 mars 2020.

45 Arrêt du Tribunal fédéral 4A_499/2019 du 25 mars 2020 c. 3.1.3. Le Tribunal fédéral ne contrôle qu'avec retenue une décision de dernière instance cantonale prise dans l'exercice du pouvoir d'appréciation, cf. Arrêt du Tribunal fédéral 4A_499/2019 du 25 mars 2020 c. 3.2.

46 Arrêt du Tribunal fédéral 4A_499/2019 du 25 mars 2020 c. 3.1.3.

47 En lien avec la levée de la situation de blocage et le but de rétablissement de la situation conforme à la loi, le Tribunal fédéral a jugé dans un autre arrêt que la procédure de carence ne s'applique que lorsqu'une disposition légale impérative concernant l'organisation de la société a été violée et vise à rétablir la situation conforme à la loi. Ellen'a en revanche pas pour objet de remettre en cause le bien-fondé économique des décisions commerciales prises par les organes, d'autres moyens, comme l'action en responsabilité, pouvant être mis en œuvre à cette fin, cf. Arrêt du Tribunal fédéral 4A_412/2020 du 16 septembre 2020 c. 4.3.4. Sur cet arrêt, voir : OLIVER DALLA PALMA / HANS CASPAR VON DER CRONE, Der Organisationsmangel in der Aktiengesellschaft und die Ernennung eines Sachwalters nach Art.731b OR, RSDA 2020, p. 577 ss. Dans cet arrêt, le Tribunal fédéral a par ailleurs exposé que la poursuite, par les administrateurs, d'intérêts opposés à ceux de la société pouvait exceptionnellement se miner les fautes, responsabilités ou mérites des actionnaires ni de dédommager ou de récompenser l'un d'eux ${ }^{48}$. Un actionnaire ne saurait revendiquer le droit d'acquérir les actions d'un autre actionnaire sur cette base. In casu, le blocage existe depuis de nombreuses années et une vente aux enchères privées des actions avec prix de réserve - dans la mesure où elle requiert une expertise prolongerait de manière non négligeable la situation de carence. La solution privilégiée par le juge respecte donc le principe de la proportionnalité ${ }^{49}$. Cette conclusion est soutenue par le fait que les sociétés n'exercent plus d'activité depuis 2015, qu'elles se trouvent dans un état de liquidation avancé (les intérêts des tiers ne sont pas lésés), et que les SA, par l'intermédiaire du commissaire, sont favorables à la liquidation. La dissolution des sociétés assure également l'égalité de traitement entre les actionnaires : en cas de vente aux enchères avec offre libre (à la place d'enchères avec prix de réserve), le risque que l'actionnaire disposant des ressources financières les plus importantes acquière les actions à un prix nettement inférieur à leur valeur réelle ne saurait être écarté50.

Le Tribunal fédéral précise qu'en cas de blocage insur- 26 montable résultant d'un conflit entre les actionnaires, une demande de dissolution fondée sur l'art. $731 \mathrm{~b}$ al. $1 \mathrm{CO}$ ne saurait servir d'instrument à l'un d'entre eux en vue d'obtenir la dissolution d'une société sans que les conditions de l'action en dissolution pour justes motifs de l'art. 736 ch. 4 CO ne soient également réunies ${ }^{51}$. Tel n'est pas le cas en l'espèce, puisque, d'une part, la procédure de carence a été initiée par le RC et que, d'autre part, le conflit insurmontable opposant les deux actionnaires est susceptible de justifier une dissolution des SA pour juste motif ${ }^{52}$. Le recours est rejeté53.

matérialiser en une situation de carence, à condition toutefois que le requérant apporte avec précision la preuve d'un tel conflit d'intérêts (c. 4.3.2). A cet égard, Le Tribunal fédéral a jugé qu'un conflit entre les deux seuls administrateurs d'une SA conduisant à une situation de blocage justifie la nomination d'un commissaire, cf. Arrêt du Tribunal fédéral 4A_380/2020 du 25 août 2020 c. 5 et 6 .

48 Arrêt du Tribunal fédéral 4A_499/2019 du 25 mars 2020 c. 4.3.

49 De même, le Tribunal fédéral a considéré dans un autre arrêt que la dissolution de la SA pour carence apparaît comme une mesure proportionnée lorsque le tribunal fixe à la SA un délai pour remédier à la carence et que les décisions nécessaires à cette fin auraient pu être prise dans le cadre d'une assemblée universelle (art. $701 \mathrm{CO}$ ). Le juge doit pallier les défauts d'organisation auxquels la SA n'est pas en mesure de remédier de manière indépendante, non parer aux défauts d'organisations que la société, respectivement les actionnaires, peuvent résoudre, $c f$. Arrêt du Tribunal fédéral 4A_439/2020 du 5 octobre 2020 c. 4.3 , 4.4. Sur cet arrêt, voir : THIEMO STURNY / DZEVRIJE ZENDELI, Gesetzgebung und Rechtsprechung, RR-VR 6/2020, p. 11.

50 Arrêt du Tribunal fédéral 4A_499/2019 du 25 mars 2020 c. 4.2 et 4.3.

51 Arrêt du Tribunal fédéral 4A_499/2019 du 25 mars 2020 c. 5.1. Le Tribunal fédéral rappelle à cet égard que le blocage des organes de la société peut constituer un juste motif au sens de l'art. 736 ch. 4 CO (c. 5.2).

52 Arrêt du Tribunal fédéral 4A_499/2019 du 25mars 2020 c. 5.3.

53 Arrêt du Tribunal fédéral 4A_499/2019 du 25 mars 2020 c. 6. 


\section{d) Qualité pour déposer une plainte $\mathbf{L P}$ du membre du CA d'une société faillie ${ }^{54}$ \\ aa) Faits} des faillites du canton de Fribourg. Il requiert la constatation de la nullité de la vente, par l'office, d'actifs de D. SA en faillite, dont il est l'administrateur unique, ainsi que la révocation de la faillite de la SA. Le 10 avril 2019, le Kantonsgericht du canton de Fribourg rejette la plainte retenant un défaut de qualité pour porter plainte. B. recourt en matière civile au Tribunal fédéral.

\section{bb) Droit}

Le Tribunal fédéral examine si B., en qualité d'organe de la société faillie, à la capacité de déposer une plainte en son propre nom, mais dans l'intérêt du failli (Prozessstandschaft), ou s'il doit agir au nom du failli. Suivant l'art. 740 al. $5 \mathrm{CO}$, les organes ne conservent le pouvoir de représenter la SA en faillite que si leur intervention est encore nécessaire ${ }^{55}$. Cet article ne modifie pas la manière par laquelle le CA agit au nom du failli. Il n'y a ni nécessité ni possibilité d'une action du CA en son nom propre en vue de défendre les intérêts d'un tiers (in casu D. SA en faillite). Si le CA souhaite déposer une plainte dans l'intérêt du failli, il doit indiquer de manière claire et précise qu'il agit en qualité d'organe et non en son nom propre. Il n'est en effet pas exclu qu'un administrateur souhaite déposer une plainte en son nom propre, et que son intérêt à agir découle précisément de sa qualité de membre du CA de la faillie ${ }^{56}$. B. ne démontrant pas qu'il aurait déposé plainte en qualité d'organe de D.SA en liquidation ${ }^{57}$, le Tribunal fédéral rejette le recours ${ }^{58}$.

\section{e) Convocation d'une AG extraordinaire, degré de la preuve ${ }^{59}$}

29 Le degré de la vraisemblance («Beweismass der Glaubhaftmachung») suffit s'agissant de la preuve du statut d'actionnaire de la personne requérant la convocation d'une AG au sens de l'art. 699 al. 4 CO60.

\footnotetext{
54 Arrêt du Tribunal fédéral 5A_375/2019 du 16 avril 2020.

55 Arrêt du Tribunal fédéral 5A_375/2019 du 16 avril 2020 c. 3.3.1.

56 Arrêt du Tribunal fédéral 5A_375/2019 du 16 avril 2020 c. 3.3.2.

57 Arrêt du Tribunal fédéral 5A_375/2019 du 16 avril 2020 c. 3.3.3.2.

58 Arrêt du Tribunal fédéral 5A_375/2019 du 16avril 2020 c. 5.

59 Arrêt du Tribunal fédéral 4A 134/2020 du 15juin 2020. A la suite d'une procédure de révision, cet arrêt a été annulé sur la base de l'art.123 al. 2 let. A du loi sur le Tribunal fédéral du 17juin 2005(LTF ; RS 173.110), cf. Arrêt du Tribunal fédéral 4F 7/2020 du 22 février 2021 (destiné à publication)

60 Arrêt du Tribunal fédéral 4A_134/2020 du 15juin 2020 c.3.3.Cf. également ATF 102 Ia 209 c. 2 ; Arrêt du Tribunal fédéral 4A 184/2019 du 15juillet 2019 c. 2.1 .
}

f) Versement indu de dividendes; rapport entre gestion d'affaires et restitution de prestations ${ }^{61}$

aa) Faits

B. Holding SA est actionnaire majoritaire de C. SA. Le 30 $1^{\text {er }}$ Octobre 2007, A. signe un contrat de travail avec C. SA. Selon le contrat, A. reçoit 340 actions de C. SA pour un montant de CHF 34'O0o.- et, en cas de résiliation, C. SA se réserve le droit de racheter les actions. La qualité d'actionnaire de A. a précédemment été confirmée par le Tribunal fédéral ${ }^{2}$. Le 13 octobre 2009, C. SA résilie le contrat de travail de A. et, le 13 décembre 2012, exerce le droit de rachat des actions de A. pour le compte de B. Holding SA (dont la légitimation pour exercer ce droit n'est pas contestée). Le prix de vente calculé par C. SA est contesté par A., qui refuse de transférer les actions. Le 28 avril 2014, A. actionne B. Holding SA en paiement des dividendes qu'il aurait dû percevoir en tant qu'actionnaire de C. SA pour les exercices 2009 à 2014. Le 23juillet 2018, le Tribunal de première instance de Genève condamne A. à céder les actions à B. Holding SA et condamne cette dernière au paiement des dividendes à A. Ce jugement est confirmé le 20 juin 2019 par la Chambre civile de la Cour de justice. B. Holding SA recourt en matière civile auprès du Tribunal fédéral.

\section{bb) Droit}

A. peut exercer l'action en remise de gain de l'art. 42331 $\mathrm{CO}-$ applicable en cas de gestion d'affaires imparfaite de mauvaise foi - pour faire valoir son droit aux dividendes pour les exercices 2009 à 2014. Cette action suppose : (1) une atteinte illicite aux droits réels ou aux droits patrimoniaux d'autrui; (2) la volonté du gérant de gérer l'affaire exclusivement ou de manière prépondérante dans son propre intérêt et (3) la mauvaise foi du gérant ${ }^{63}$. In casu, (1) B. Holding SA a perçu indûment les dividendes auxquels A. avait droit pour les exercices 2009 à 2014 ; (2) la société a eu la volonté d'encaisser les dividendes dus à A. exclusivement dans son propre intérêt et (3) B. Holding SA ne pouvait pas de bonne foi ignorer que A. était actionnaire ${ }^{64}$

L'action en remise de gain basée sur l'art. 423 CO ne sau- 32 rait être exclue par le fait que A. pourrait agir contre $\mathrm{B}$. Holding SA par le biais de l'action sociale oblique en restitution (art. $678 \mathrm{al} .3 \mathrm{CO}$ ). Les deux actions reposent sur

61 Arrêt du Tribunal fédéral 4A_450/2019, 4A_460/2019 du18 mai 2020. 62 Arrêt du Tribunal fédéral 4A 646/2014 du 14 avril 2015 et Arrêt du Tribunal fédéral 4A_248/2017 du 22 février 2018. Sur le second arrêt, cf. DAMIANO CANAPA / ARTHUR GRISONI / LINE ELODIE DERUNGS, Le droit des sociétés en 2018: Modifications législatives, jurisprudence et doctrine, sui generis 2018, p. $310 \mathrm{s.}$

63 Arrêt du Tribunal fédéral 4A_450/2019,4A_460/2019 du18 mai 2020 c. 5.1.1.

64 Arrêt du Tribunal fédéral 4A_450/2019,4A_460/2019 du18 mai 2020 c. 5.2 . 
des fondements juridiques distincts et sont indépendantes l'une de l'autre : l'actionnaire qui intente une action en restitution doit conclure au paiement en mains de la société, alors que l'action en remise de gain vise à protéger directement le maître qui subit une atteinte illicite à ses droits. Il n'y a aucune raison d'empêcher A. (le maître) de faire valoir ses prétentions contre B. Holding SA en agissant directement contre cette dernière par l'action en remise de gain ${ }^{65}$.

Enfin, dans la mesure où le capital-actions de C. SA est constitué exclusivement d'actions au porteur, le transfert des seuls droits économiques - en particulier du droit aux dividendes -, à l'exclusion des droits sociaux, n'est pas envisageable (principe de l'unité de l'action): A. est demeuré propriétaire des actions après l'exercice du droit de rachat par B. Holding SA le 13 décembre 2012; cette dernière ne saurait invoquer qu'un contrat de vente inconditionnel aurait été conclu au moment de l'exercice du droit de rachat ayant pour effet de lui transférer les profits et les risques liés aux actions ${ }^{66}$. Le recours est rejeté67.

\section{g) Principe de la transparence ${ }^{68}$ \\ aa) Faits}

En juin 2016, B. est devenu l'actionnaire unique de C. SA. Depuis le 17 octobre 2017, B. dispose d'un droit de signature individuelle. Le 30 mai 2016, une procuration avait été conférée par C. SA à l'avocat A., afin qu'il assiste C. SA dans la procédure de faillite ouverte contre elle. Les 12 août et 3 novembre 2016, A. fait parvenir à B. deux notes d'honoraires ; ces notes n'ont pas été acquittées. A. recourt au Tribunal fédéral contre l'arrêt de la Cour de justice du canton de Genève qui condamne C. SA, à l'exclusion de B., au versement des honoraires en invoquant une violation du principe de la transparence.

\section{bb) Droit}

'application du principe de la transparence (Durchgriff) suppose (1) une identité de personnes conformément à la réalité économique (ou au moins la domination économique d'un sujet de droit sur l'autre) et (2) une invocation de la dualité des personnes constitutive d'un abus de droit ou ayant pour effet une atteinte manifeste à des intérêts légitimes, pour en tirer un avantage injustifié (art.2 al.2 CC) ${ }^{69}$. Le Tribunal fédéral rejette l'application

65 Arrêt du Tribunal fédéral 4A_450/2019, 4A_460/2019 du 18 mai 2020 c. 5.3 .2 .

66 Arrêt du Tribunal fédéral 4A_450/2019, 4A_460/2019 du 18 mai 2020 c. 5.3.5.

67 Arrêt du Tribunal fédéral 4A_450/2019, 4A_460/2019 du 18 mai 2020 c. 6 .

68 Arrêt du Tribunal fédéral 4A_600/2019 du 17juin 2020.

69 Arrêt du Tribunal fédéral 4A_600/2019 du 17juin 2020 c. 4.1. du principe et par conséquent le recours ${ }^{70}$ : au moment de la signature de la procuration émise pour C. SA, B. n'était ni l'actionnaire unique ni ne disposait à l'égard de la société d'un droit de signature individuelle ${ }^{71}$.

\section{h) Représentation 72}

Les dispositions sur la représentation de la SA - «repré- 36 sentation » par les organes (art. 718 al. 1 et 2 CO) et représentation commerciale (art. 721 cum 458 et 462 CO) - ne font pas obstacle à une représentation de la société par des représentants civils au sens des art. 32 ss CO${ }^{73}$.

\section{i) Nullité des décisions de l'AG et du CA - intérêt à agir ${ }^{74}$}

aa) Faits

B. SA, C. SA, D. SA, E. SA et F. SA sont des sociétés appa- 37 rentées. Le 8 février 2016, les CA et AG des différentes sociétés votent les dissolutions respectives de ces dernières. Le 15 février 2017, A. - actionnaire de F. SA et administratrice de B. SA, C. SA, D. SA et E. SA - intente une action en constatation de la nullité des décisions du 8 février 2016 auprès du Kantonsgericht du canton de Zoug. Les décisions de dissolution sont révoquées les 13 avril et 31 juillet 2018. Le 8 novembre 2018, l'action de A. est rejetée faute d'intérêt à agir digne de protection, jugement qui est confirmé par l'Obergericht du même canton le 6juin 2019. A. recourt matière civile.

\section{bb) Droit}

En droit des sociétés, la notion d'intérêt digne de protec- 38 tion à agir en constatation de droit (art. 88 CPC) s'apprécie de manière large : un tel intérêt existe lorsqu'une action tend à protéger les intérêts de la société, sous réserve de l'interdiction de l'abus de droit ${ }^{75}$. Tel est notamment le cas lorsque les rapports juridiques entre les parties sont incertains et que cette incertitude - qui entrave la liberté d'action du demandeur et ne peut être tolérée par ce dernier - peut être levée par une décision en constatation ${ }^{76}$.

Les sociétés n'étaient en tout cas plus en liquidation de- 39 puis le moment de la révocation des décisions de dissolution (que ces dernières aient été nulles ab initio ou non);

\section{Arrêt du Tribunal fédéral 4A_600/2019 du 17juin 2020 c. 5 . \\ 71 Arrêt du Tribunal fédéral 4A_600/2019 du 17juin 2020 c. 4.2. \\ 72 Arrêt du Tribunal fédéral 4A 562/2019 du 10 juillet 2020. Sur cet ar- rêt, voir aussi PETER REETZ et al., BR 2020, p. $348 \mathrm{~s}$. \\ 73 Arrêt du Tribunal fédéral 4A 562/2019 du 10 juillet 2020 c. 4. Sur les différents modes de représentation des corporations, cf. ATF 146 III 37; Arrêt du Tribunal fédéral 4A 187/2018 du 21 février 2019 c. 3. Pour un résumé de ces arrêts, voir DAMIANO CANAPA / ARTHUR GRISONI / LINE ELODIE DERUNGS, Le droit suisse des sociétés en 2019: modi- fications législatives, jurisprudence et doctrine, sui generis 2020, p. 429 ss.}

74 Arrêt du Tribunal fédéral 4A_282/2020 du 5août 2020.

75 Arrêt du Tribunal fédéral 4A_282/2020 du 5août 2020 c. 2.1.

76 Arrêt du Tribunal fédéral 4A_282/2020 du 5 août 2020 c. 2.2. 
l'incertitude des rapports juridiques ne peut donc concerner que la période comprise entre le moment où les décisions de dissolution ont été prises et celui où elles ont été révoquées ${ }^{77}$. A. n'explique pas en quoi une décision rendue dans une action constatatoire (qui a un caractère subsidiaire) lui conférerait, à elle ou aux sociétés, un avantage concret qui ne pourrait être obtenu par une action condamnatoire ou formatrice ${ }^{78}$. Elle ne démontre pas non plus l'existence d'un intérêt actuel et indépendant à faire constater la nullité des décisions du 8 février 2016, qui se rapportent à des événements passés et clôturés. Si A. estimait que des actes de liquidation ont causé un préjudice aux sociétés, elle aurait dû ouvrir une action condamnatoire (telle qu'une action en responsabilité); dans ce cadre, elle aurait dû soulever la nullité des décisions de dissolution sur lesquelles se seraient fondés les actes de liquidation ${ }^{79}$. Le Tribunal fédéral rejette le recours ${ }^{80}$.

\section{j) Annulation et nullité des décisions de l'AG - légitimitéactive et lien de causalité ${ }^{81}$}

aa) Faits

40 M.A. et D.A. acquièrent par succession 25 des 50 actions de X. SA et de Y. SA. Le partage n'étant pas encore intervenu, ces actions sont la propriété commune des deux héritiers. M.A. est propriétaire des 25 actions restantes. Le 27 mai 2014, les deux sociétés tiennent leurs AG. Au terme de celles-ci, D.A. est révoqué de son mandat d'administrateur des deux sociétés, sans que la révocation d'un administrateur n'ait figuré sur les ordres du jour. D.A. ouvre action en constatation de la nullité des décisions révoquant ses mandats d'administrateur devant le Tribunal de première instance du canton de Genève, qui le déboute. Après le rejet de son appel par la Cour de justice genevoise ${ }^{82}$, D.A. recourt en matière civile au Tribunal fédéral.

\section{bb) Droit}

e Tribunal fédéral, un vice de procédure formel tel qu'une convocation de l'AG ne comprenant pas un ordre du jour complet - ne peut entraîner la nullité d'une

\footnotetext{
77 Arrêt du Tribunal fédéral 4A_282/2020 du 5 août 2020 c. 4.1, 4.2. La requérante ne prétend d'ailleurs pas qu'au moment de la révocation des décisions de dissolution, la distribution des actifs avait déjà commencé.

78 Arrêt du Tribunal fédéral 4A_282/2020 du 5août 2020 c. 4.2.1.

79 Arrêt du Tribunal fédéral 4A_282/2020 du 5 août 2020 c. 4.2.2.

80 Arrêt du Tribunal fédéral 4A_282/2020 du 5août 2020 c. 5.

81 Arrêt du Tribunal fédéral 4A_141/2020 du 4 septembre 2020.

82 Dans le cadre d'une procédure antérieure, le Tribunal fédéral a jugé que le D.A. ne disposait pas seul de la qualité pour agir en annulation puisqu'ilétait propriétaire en main commune des actions avec M.A. et qu'aucun représentant commun n'avait été désigné (consorité matérielle nécessaire), cf. Arrêt du Tribunal fédéral 4A_516/2016 du 28 août 2017 c. 7 et 8 . Pour un résumé de cet arrêt, voir DAMIANO CANAPA / MARLEEN BOTTERBRODT / ANTOINE SCHNEEBELI, Le droit suisse des sociétés en 2017: modifications législatives, jurisprudence et doctrine, sui generis 2018, p. 308.
}

décision (art. 706 $b \mathrm{CO}$ ) que s'il existe un lien de causalité entre le vice invoqué et le contenu de la décision. Il convient de prouver que le déroulement sans vice de la procédure aurait abouti à une décision (hypothétique) différente ${ }^{83}$. En l'espèce, D.A. n’a pas démontré qu'il aurait été en mesure de convaincre M.A ou un éventuel représentant commun (art. 690 CO) de s'opposer à la révocation de son mandat d'administrateur si cette dernière avait été portée à l'ordre du jour. Le vice de procédure formel soulevé ne saurait donc entraîner la nullité de la décision de l'AG ${ }^{84}$. Le Tribunal fédéral rejette le recours ${ }^{85}$.

\section{k) Violation des devoirs de diligence et de fidélité et de l'obligation de renseigner du président du $\mathrm{CA}^{86}$}

\section{aa) Faits}

La banque privée A. SA est détenue indirectement par 42 A.I. SA, qui est contrôlée par le groupe A. Le 17 septembre 2014, à la suite de difficultés financières de A.I. SA, la FINMA prononce la faillite de la banque A. SA en raison de son surendettement et du non-respect des prescriptions en matière de fonds propres. Le 8 juillet 2016, à l'issue d'une procédure d'enforcement ouverte pour soupçon de violation du droit de la surveillance (art. 30 LFINMA ${ }^{87}$ ), la FINMA prononce à l'encontre de A.A. - ancien président du CA de la banque A. SA et membre avec droit de vote du conseil supérieur du groupe A. - une interdiction d'exercer toute fonction dirigeante dans l'établissement d'un assujetti à la FINMA pour une durée de cinq ans (art. 33 LFINMA). Il lui est reproché de ne pas avoir immédiatement informé $\mathrm{A}$. SA de la situation financière critique de A.I. SA - qui se trouvait en situation de surendettement dès la fin de l'année 2013 - ou, à tout le moins, de ne pas avoir annoncé à A. SA l'existence d'un conflit d'intérêts concret et important entre ses différents mandats au sein du groupe A. Saisi du recours formé par A.A., le TAF confirme la décision de la FINMA. A.A. recourt en matière de droit public au Tribunal fédéral.

\section{bb) Droit}

En tant qu'administrateur de A. SA, A.A. avait le devoir 43 d'exercer ses attributions intransmissibles (art. $716 \mathrm{a} \mathrm{CO}$ ) avec toute la diligence requise (art. $717 \mathrm{CO}$ ). Un administrateur - membre ou non du comité d'audit ou des finances - doit suivre personnellement et avec circonspection l'évolution financière et économique de la SA88. Conformément à son devoir de fidélité (art. 717 CO), A.A.

83 Arrêt du Tribunal fédéral 4A_141/2020 du 4 septembre 2020 c. 3.2. 84 Arrêt du Tribunal fédéral 4A_141/2020 du 4 septembre 2020 c. 3.4.2. 85 Arrêt du Tribunal fédéral 4A_141/2020 du 4 septembre 2020 c. 4. 86 Arrêt du Tribunal fédéral 2C_771/2019 du 14 septembre 2020.

87 Loi sur l'Autorité fédérale de surveillance des marchés financiers du 22juin 2007 (Loi sur la surveillance des marchés financiers, LFINMA; RS 956.1).

88 Arrêt du Tribunal fédéral 2C_771/2019 du 14 septembre 2020 c. 19.2.1. 
devait subordonner ses comportements aux intérêts de la SA et prévenir tout risque de conflit avec ses intérêts propres, ceux des actionnaires ou de tiers ; la collision des devoirs de fidélité résultant de la fonction d'organe au sein de plusieurs sociétés ne saurait être considérée comme un motif justificatif ${ }^{89}$. La mesure de la diligence requise dépend des circonstances et il faut se demander si, sur la base des renseignements dont disposait ou devrait disposer l'administrateur, l'attitude de ce dernier parait raisonnablement défendable ${ }^{90}$. Enfin, le président du CA doit adopter un comportement proactif en lien avec le droit aux renseignements des administrateurs (art. 715 $a$ CO) : il doit prendre l'initiative de transmettre au CA les informations pertinentes pour l'exercice de ses tâches (tels que des événements extraordinaires d'importance majeure) ${ }^{91}$.

In casu, A.A., en qualité d'organe du groupe A., de la banque, de A.I. SA et de membre du conseil supérieur avec droit de vote, connaissait le risque de surendettement de A.I. SA. Il n'a entrepris aucune démarche à temps afin d'en informer A. SA, dont il présidait le CA. De par sa fonction, il avait pourtant un devoir accru d'informer les administrateurs afin qu'ils puissent exercer effectivement leur droit aux renseignements et que le CA in globo puisse prendre les mesures qui s'imposent. En conséquence, A.A. a violé ses devoirs de diligence et de fidélité et son obligation d'informer ${ }^{92}$; cela illustre le conflit d'intérêts auquel A.A. était confronté, en devant respecter les intérêts des différentes sociétés dans lesquelles il siégeait, en particulier ceux de A.I. SA ${ }^{93}$. Le comportement fautif de A.A. étant en lien de causalité direct avec les violations graves du droit de la surveillance commises par la banque (violation des devoirs d'organisation et de gestion des risques, art. 3 al. 2 let. a LB94, art. 9 al. 2 aOB95 ; violation du devoir d'annonce, art. 29 al. 2 LFINMA $\left.{ }^{96}\right)^{97}$, les conditions d'application de l'art. 33 LFINMA sont remplies. Le Tribunal fédéral rejette le recours ${ }^{98}$.

89 Arrêt du Tribunal fédéral 2C_771/2019 du 14 septembre 2020 c. 19.2.2. 90 Arrêt du Tribunal fédéral 2C_771/2019 du 14 septembre 2020 c. 19.2.1. 91 Arrêt du Tribunal fédéral 2C_771/2019 du 14 septembre 2020 c. 19.2.3.

92 Arrêt du Tribunal fédéral 2C_771/2019 du14 septembre 2020 c. 19.3 et 19.4 .

93 Arrêt du Tribunal fédéral 2C 771/2019 du 14 septembre 2020 c. 19.4 . Le Tribunal fédéral relève que le fait de siéger dans plusieurs sociétés d'un même groupe n'est pas contraire au droit et ne conduit pas ispofacto à des difficultés avec le devoir de fidélité: le recourant ne saurait tirer d'argument du fait que ni le réviseur ni la FINMA n'avaient émis de critiques en lien avec les différentes fonctions exercées au sein du groupe.

94 Loi fédérale sur les banques et les caisses d'épargne du 8 novembre 1934 (Loi sur les banques, LB; RS 952.0).

95 Ordonnance sur les banques et les caisses d'épargne du 12 mai 1972 (aOB; RS 952.02). Arrêt du Tribunal fédéral 2C_771/2019 du 14 septembre $2020 \mathrm{c} .17$.

96 Arrêt du Tribunal fédéral 2C $771 / 2019$ du 14 septembre 2020 c. 18.3 . 97 Arrêt du Tribunal fédéral 2C_771/2019 du 14 septembre 2020 c. 20. 98 Arrêt du Tribunal fédéral 2C_771/2019 du 14 septembre 2020 c. 24.

\section{l) Contrôle spécial ${ }^{99}$}

aa) Faits

B.B. et C.B. détiennent 67, respectivement 2, des 600 ac- 45 tions nominatives de A. SA. Lors de l'AG du 25juin 2019, C.B. demande des renseignements sur les raisons de l'augmentation conséquente des charges de personnel de la SA au cours de l'exercice 2018 par rapport à l'exercice précédent. Cette demande est réitérée par B.B. - qui est en conflit avec A. SA depuis 2016 - et C.B. lors l'AG extraordinaire du 4 novembre 2019. Ils proposent l'institution d'un contrôle spécial (art. 697a CO) afin d'élucider la question, proposition qui est rejetée par l'AG. Le 12 mai 2020, sur requête de C.B. et B.B., le Handelsgericht du canton de Berne désigne un contrôleur spécial (art. $697 \mathrm{~b}$ CO). A. SA recourt en matière civile au Tribunal fédéral.

\section{bb) Droit}

Les informations recueillies par le contrôleur spécial 46 doivent être nécessaires à l'exercice des droits des actionnaires. Pour le Tribunal fédéral, lorsqu'une distribution dissimulée de bénéfices (art. 678 al. 2 CO) prend la forme de salaires excessifs, il s'agit potentiellement d'une violation de règles matérielles réglementant la distribution de bénéfices qui ne saurait être "guérie» par l'approbation des comptes annuels par l'AG'100. Les montants des salaires sont des éléments internes à la SA, dont la connaissance par les actionnaires est nécessaire pour envisager une action en restitution (art. 678 CO), comme envisagée par les requérants ${ }^{\mathbf{1 0 1}}$. La preuve par vraisemblance s'appliquant tant aux faits qu'au droit, le Handelsgericht n'a pas violé la loi en considérant que les actionnaires requérant le contrôle spécial avaient rendu vraisemblable une violation de l'art. 678 CO ayant causé un préjudice à la société ou aux actionnaires (art. 697b al. 2 CO) ${ }^{102}$.

Le fait qu'A. SA, d'une part, et B.B. et C.B., d'autre part, 47 sont en conflit ne suffit pas à conclure que la demande d'examen spécial constituerait un abus de droit (art. 2 al. 2 CC) : la loi tient compte d'intérêts potentiellement divergents de ce type en prévoyant qu'une minorité qualifiée d'actionnaires peut requérir du juge qu'il nomme un contrôleur spécial. Dans cette mesure, un comportement abusif ne peut se déduire de la seule existence d'intérêts contradictoires ${ }^{103}$. Le recours est rejeté104.

\section{Arrêt du Tribunal fédéral 4A_312/2020 du 15 octobre 2020. \\ 100 Arrêt du Tribunal fédéral 4A_312/2020 du 15 octobre 2020 c. 3.2.2. Seule une distribution de bénéfices intervenant en violation de règles formelles peut être "guérie » par une décision a posteriori de l'AG. \\ 101 Arrêt du Tribunal fédéral 4A_312/2020 du15octobre 2020 c. 3.1,3.3.2.} 102 Arrêt du Tribunal fédéral 4A_312/2020 du 15 octobre 2020 c. 4 . 103 Arrêt du Tribunal fédéral 4A_312/2020 du 15 octobre 2020 c. 5.2. 104 Arrêt du Tribunal fédéral 4A_312/2020 du 15octobre 2020 c. 6 . 


\section{m) Action en annulation; capacité d'être partie et d'ester en justice du $\mathrm{CA}^{105}$}

aa) Faits

Par décision du 27 février 2020, l'AG de C. SA exclut A. et B. du CA. Le 21 avril 2020, ces derniers requièrent du Handelsgericht du canton de Zurich l'annulation de la décision (art. $706 \mathrm{CO}$ ) en leur nom propre et « au nom et sur mandat du CA de C. SA». Le 29 mai 2020, sur requête de C. SA, ce tribunal prononce des mesures superprovisionnelles qui interdisent à A. et B. d'agir en qualité d'administrateurs de la SA et d'accomplir tout acte juridique au nom la société ou d'agir au nom de cette dernière. Ces mesures sont confirmées le 24juin 2020 par le Handelsgericht dans un jugement sur mesures provisionnelles contre lequel A. et B. recourent en matière civile.

\section{bb) Droit}

Une décision sur mesures provisionnelles est une décision incidente qui ne peut faire l'objet d'un recours au Tribunal fédéral que si elle peut causer un préjudice irréparable (art. 93 al. 1 lit. a LTF) ${ }^{\mathbf{1 0 6}}$. In casu, ces mesures superprovisionelles ne privent pas A. et B. du droit d'intenter une action en annulation. C'est le CA, en qualité d'organe de la SA, qui a la capacité d'être partie et d'ester en justice dans les actions tendant à l'annulation des décisions de l'AG. Pour exercer ce droit, le CA doit prendre une décision. Or, une telle décision a été prise avant le prononcé des mesures superprovisionnelles. A. et B. ne violent donc pas les mesures superprovisionelles s'ils

105 Arrêt du Tribunal fédéral 4A_403/2020,4A_405/2020 du 1 ${ }^{\text {er }}$ décembre 2020.

106 Arrêt du Tribunal fédéral 4A_403/2020, 4A_405/2020 du 1 ${ }^{\text {er }}$ décembre 2020 c. 2.1 exécutent uniquement cette décision. D’une part, ils n'agissent pas en tant qu'administrateurs individuels, mais ils représentent le CA. D'autre part, A. et B. interviennent contre la SA au nom du CA, en qualité d'organe, et non au nom et en qualité de représentants de la société. En l'absence de préjudice irréparable pouvant résulter de la décision attaquée ${ }^{107}$, le recours est irrecevable ${ }^{108}$.

\section{Société à responsabilité limitée}

a) Invocation abusive d'un vice de forme ${ }^{109}$

Un contrat de prêt doit être conclu par écrit si, comme 50 dans le cas présent, la société est représentée par la personne avec laquelle elle contracte (sauf en cas d'opération courante, cf. art. 814 al. 4 cum $718 b$ CO). Pour le Tribunal fédéral, il est inadmissible d'invoquer la nullité d'un contrat pour non-respect d'une exigence de forme de manière contraire au principe de la bonne foi, par exemple en cas de comportement contradictoire; une telle situation constitue un abus de droit (art. 2 al. 2 CC). Il convient d'analyser si l'objectif poursuivi par l'exigence de forme a été violé au détriment de la partie qui invoque le vice. In casu, A. - associé unique et directeur général de la Sàrl-ne saurait invoquer la nullité du contrat de prêt pour non-respect de l'exigence de forme alors qu'il a conclu lui-même le contrat en tant que représentant de la société et que cette dernière a déjà exécuté son obligation ${ }^{110}$.

\footnotetext{
107 Arrêt du Tribunal fédéral 4A_403/2020, 4A_405/2020 du $1^{\text {er }}$ décembre 2020 c. 2.3.3.

108 Arrêt du Tribunal fédéral 4A 403/2020, 4A 405/2020 du 1er décembre 2020 c. 3.

109 Arrêt du Tribunal fédéral 4A 545/2019 du 13 février 2020

110 Arrêt du Tribunal fédéral 4A_545/2019 du 13 février 2020 c. 5.2.4.
} 